\title{
Modelling the broadband emission from the white dwarf binary system AR Scorpii
}

\author{
K K Singh ${ }^{\mathrm{a}, \mathrm{b}, *}$, P J Meintjes ${ }^{\mathrm{a}}$, Q Kaplan ${ }^{\mathrm{a}}$, F A Ramamonjisoa ${ }^{\mathrm{a}}$, \\ S Sahayanathan ${ }^{\mathrm{b}}$ \\ ${ }^{a}$ Physics Department, University of the Free State, Bloemfontein - 9300, South Africa \\ ${ }^{\mathrm{b}}$ Astrophysical Sciences Division, Bhabha Atomic Research Centre, Mumbai- 400085 , \\ India
}

\begin{abstract}
AR Scorpii is a compact binary system which consists of a magnetic white dwarf and an M-type main sequence cool star. This binary system was discovered as a source of pulsating radiation in radio, infrared, optical, ultraviolet and X-ray wavebands. In this work, we have analyzed the $\gamma$-ray data in the energy range $100 \mathrm{MeV}$ to $500 \mathrm{GeV}$ from the FermiLarge Area Telescope (LAT) observations for the period August 4, 2008 to March 31, 2019. The $\gamma$-ray emission from AR Scorpii over the last decade is not statistically significant and therefore $2 \sigma$ upper limit on the integral flux above $100 \mathrm{MeV}$ has been estimated. We reproduce the non-thermal broadband spectral energy distribution of AR Scorpii using an emission model having two synchrotron components due to the relativistic electrons in very high magnetic fields. The first component (Synchrotron-1) broadly describes the emissions at radio to high energy $\mathrm{X}$-rays through the synchrotron radiation originating from a spherical region of radius $\sim 1.8 \times 10^{10} \mathrm{~cm}$ and a magnetic field strength of $\sim 10^{3}$ Gauss. The second component (Synchrotron-2) which reproduces the X-ray emission at lower energies and predicts the $\gamma$-ray emission, originates from another spherical region with radius $\sim 1.4 \times 10^{10} \mathrm{~cm}$ and a magnetic field strength of $\sim 10^{6}$ Gauss. The relativistic electron populations in both the emission regions are described by a smooth broken power law energy distribution. The $\gamma$-ray emission predicted by the Synchrotron- 2 model is below the broadband sensitivity of the Fermi-LAT and is also consistent with the $95 \%$ confidence level upper limit on the integral flux above $100 \mathrm{MeV}$ derived from more than 10 years of observations. According to our model, the binary system AR Scorpii could be a $\gamma$-ray source, although its emission level must be below the current detection limit of the Fermi-LAT.
\end{abstract}

Key words: (binaries:) white dwarfs:individual: AR Scorpii-methods:data analysis-Gamma-rays: general-radiation mechanisms:non-thermal

\footnotetext{
* Corresponding author.

Email address: kksastro@barc.gov. in (K K Singh).
} 


\section{Introduction}

White dwarfs (WDs) are formed as the end products of the evolution of intermediate main sequence stars in the cores of red giant stars [1,2]. They are considered as compact objects (electron-degenerate stellar configurations) with an average mass of $\sim 0.6 \mathrm{M}_{\odot}$ and radius of the order of $10^{8} \mathrm{~cm}(\sim$ Earth-size $)$. WDs have therefore a very high average density of $10^{6} \mathrm{~g} \mathrm{~cm}^{-3}$ and a large surface gravity. They are found as isolated objects or companions in various astrophysical binary systems with compact objects (black hole, neutron star, or white dwarf), main sequence stars, giant stars or ordinary stars. Isolated WDs are observed with very high surface magnetic fields in the range $10^{3}-10^{9}$ Gauss [3]. They are referred to as magnetic white dwarfs (MWDs) and emit at ultraviolet and near-infrared wavelengths. The mass of MWDs $\left(\sim 0.8 \mathrm{M}_{\odot}\right)$ is more than the mass of non-magnetic or weaklymagnetic WDs $\left(\sim 0.5 \mathrm{M}_{\odot}\right)$ [3]. MWDs also exist in binary systems where they strip or accrete material from a nearby companion star. For main sequence companion stars, the accretion mainly occurs through Roche-lobe overflow whereas it is driven by the stellar wind in the case of red giant stars [4]. The resulting mass transfer from the main sequence companion stars to the MWDs produces atomic lines and X-ray emissions from the MWD binary systems [5,6]. In a typical WD binary system, the accreted matter heats up and forms a shock wave before settling down on the surface of the WD. Under the assumption of a dipole magnetic field, a MWD would behave like a radio pulsar [7]. The radio pulsars like Crab, characterized as the fast-rotating magnetized neutron stars, are assumed to be powered by the spin-down energy loss due to the rotation or spinning of neutron star [8]. The spin-down luminosity or rotational energy loss $\left(L_{\text {down }}\right)$ of the pulsar or MWD is given by [9]

$$
L_{\text {down }}=\frac{8 \pi^{2} M R^{2} \dot{P}}{5 P^{3}}
$$

where $M$ and $R$ are the mass and radius of the WD respectively which are used to define the moment of inertia as $I=\frac{2}{5} M R^{2} . P$ and $\dot{P}$ are the spin period and its time derivative respectively. The magnetic spin-down luminosity $\left(L_{\text {mag }}\right)$ for a magnetic dipole approximation of the MWD and assuming a wind outflow from the magnetosphere, can be approximated as [10]

$$
L_{m a g}=\frac{16 \pi^{4} B_{s}^{2} R^{6}}{6 c^{3} P^{4}}
$$

where $B_{s}$ is the strength of the surface magnetic field at the polar cap and $c$ is the speed of light in vacuum. If the magnetic dipole losses of the WD dominate the luminosity of a binary system, the strength of the surface magnetic field of the MWD at the polar cap is related to the different parameters of the pulsar as [10]

$$
B_{s}=\sqrt{\frac{3 M c^{3} P \dot{P}}{5 \pi^{2} R^{4}}}
$$


and the light cylinder radius $\left(R_{L C}\right)$ of the spinning MWD is defined by

$$
R_{L C}=\frac{c P}{2 \pi}
$$

The magnetic field strength at the light cylinder $\left(B_{L C}\right)$ is approximated as

$$
B_{L C}=4 \pi^{2} \sqrt{\frac{3 M R^{2} \dot{P}}{5 c^{3} P^{5}}}
$$

Therefore, a precise measurement of $P$ and $\dot{P}$ is very important to derive the energetics of a pulsar or spinning MWD. $B_{L C}$ plays a very crucial role for the $\gamma$-ray emission from these sources due to the conversion of a significant fraction of the spin-down power $\left(L_{\text {down }}\right)$ to $\gamma$-ray luminosity.

The binary systems comprising the MWDs and main sequence stars are divided into two classes namely polar and intermediate polar depending on the strength of the surface magnetic field of the WD. For polar type, the surface magnetic field $\left(B_{s}\right)$ is higher than $10^{7}$ Gauss and accretion from the companion star is completely confined through the magnetic field of the WD [11]. In the case of intermediate polar class, $B_{s}$ is in the range $10^{3}-10^{6}$ Gauss and the accretion process is partially channeled through the surface magnetic field [12]. Due to the stronger surface magnetic fields, the rotation of the two stars is synchronised in the polar binary systems. Whereas, in the intermediate polar systems, the rotation of the WD is not synchronised with the companion star and the spin period of the WD is shorter than the orbital period. The formation of an accretion disk around the WD is prevented in the polar binary systems because of the synchronised rotation of the two stars and no angular momentum of the accreting matter with respect to the WD. But, an accretion disk can be formed in the intermediate polar systems and the transfer of angular momentum from the accreting matter may spin up the WD [13]. The accretion flows in polars and intermediate polars lead to the formation of funnels and curtains respectively. The differential rotation of gaseous matter leaking out from the companion star along a circular Keplerian orbit leads to a viscous rubbing of fluid elements at varying distances and causes the accretion disk to heat up. A sufficiently hot accretion disk emits radiation from optical, ultraviolet to X-rays. The surface magnetic field in the intermediate polar disrupts the inner part of the accretion disk and the accreting matter along the magnetic field lines [13]. This leads to the formation of one or more accretion columns near the magnetic poles which emit non-thermal polarized radiation from radio to X-rays. The variations in the non-thermal emissions from the intermediate polar binary systems are strongly modulated on the spin period $(P)$ of the MWD and its harmonics [14]. However, the exact physical process involved in the non-thermal broadband emission from the WD binary systems has not been clearly understood and is being widely debated. In this work, we model the time averaged broadband spectral energy distribution (SED) of an intermediate polar AR Scorpii using observations spanned over last two decades. In Section 2, we present a brief description of the important 
observational features so far of AR Scorpii. The details of the broadband data set used in the present work are given in Section 3. In Section 4, we discuss the results from the multi-wavelength emission from the binary system AR Scorpii. Finally, we conclude the study in Section 5.

\section{AR Scorpii}

AR Scorpii is an intermediate polar type WD binary system at a distance of $d \sim$ $110 \mathrm{pc}\left(3.4 \times 10^{20} \mathrm{~cm}\right)$ from the Earth and is located in the ecliptic plane near to the Galactic centre [14]. It consists of an MWD and an M-type main sequence companion star with a binary separation of $a \sim 7.6 \times 10^{10} \mathrm{~cm}$ and both move around the common centre of mass. The mass and radius of the MWD in AR Scorpii are $M=0.8 M_{\odot}$ and $R=7 \times 10^{8} \mathrm{~cm}$ respectively and the surface magnetic field $B_{s} \sim 10^{8}$ Gauss [14]. The companion M-star has a mass of $m \sim 0.3 M_{\odot}$ and a radius of $r \sim 2.7 \times 10^{10} \mathrm{~cm}$. In early observations during 1970-71, AR Scorpii was classified as a periodic variable star $(\delta$-Scuti) and recently it was discovered as a close MWD binary system with an orbital period of $P_{o} \sim 3.56$ hours and a spin period of the MWD $P \sim 1.95$ minutes (117 seconds) at radio and optical wavelengths [14]. The measured time derivative of the spin period of the MWD in AR Scorpii was $\dot{P} \sim 3.9 \times 10^{-13}$ seconds/second. This gives an estimate of the age of the MWD as $t=\frac{P}{2 \dot{P}} \sim 4.5 \times 10^{6}$ years. The photometry of AR Scorpii taken over seven years suggests that the R-band optical magnitude changes from 16.9 (faintest) to 13.6 (brightest) on a time period of $\sim 3.56$ hours. The amplitude spectra corresponding to the ultraviolet, optical, infrared and radio $(9 \mathrm{GHz})$ fluxes show signals having two components, which are identified as the spin frequency $\left(\nu_{s}=P^{-1}\right)$ and beat frequency $\left(\nu_{b}=\nu_{s}-\nu_{o}\right.$, where $\nu_{o}=P_{o}^{-1}$ is the orbital frequency). The beat component of the signal is stronger and therefore it defines the dominant pulsation at a beat period of $P_{b}=1.97$ minutes and its harmonics [14]. The observations of AR Scorpii over a wide wavelength range from radio to ultraviolet show strong doublehumped pulsations with the pulse fractions between $10 \%$ to $95 \%$ [14]. The optical spectra of M-type cool main sequence star with absorption lines suggest that the radial velocity of the companion star in AR Scorpii varies sinusoidally with a time period of $\sim 3.56$ hours and an amplitude of $\sim 290 \mathrm{~km} \mathrm{~s}^{-1}$ [14]. The MWD in AR Scorpii is not visible in the optical spectra. For a spin period $P \sim 117$ seconds, the light cylinder radius of the MWD $R_{L C} \approx 5.6 \times 10^{11} \mathrm{~cm}$ (from Equation 4) which is about seven times longer than the binary separation. This implies that the companion star in AR Scorpii is situated well inside the magnetosphere of the MWD. The MWD in AR Scorpii is spinning down on a characteristic timescale of $\sim 10^{7}$ years [14]. The X-ray emission from AR Scorpii is significantly modulated over the spin period of the MWD with a pulse fraction of $\sim 14 \%$ [15]. The maximum or minimum X-ray intensity is located at the superior or inferior conjunction of the M-star orbit. An evidence of a power law spectrum is found in the pulsed compo- 
nent of the X-ray emission [15]. The observed optical emission from AR Scorpii modulating with the beat frequency of the binary system is highly polarized with a degree of linear polarization up to $40 \%$ and a low level of circular polarization of less than $5 \%[16,17]$. The degree of linear polarization evolves with the orbital phase and varies on both the beat frequency of the binary system and spin frequency of the MWD. The observed characteristics of the degree of linear polarization are similar to that of the well-known spin-powered Crab pulsar. The radio observations at $8.5 \mathrm{GHz}$ reveal the compactness of the emission region and confirm the identity of AR Scorpii as a point Galactic source [18]. The high resolution interferometric radio imaging of AR Scorpii indicates a strong modulation of the radio flux on the orbital and beat periods and the radio emission exhibits a weak linear polarization and very strong circular polarization [19]. High temporal resolution spectroscopy of this MWD binary system shows a complex structure of the $H \alpha$ emission lines as observed from some polars in the quiescent state [20]. The aforementioned measurements/results suggest a non-thermal origin of the broadband radiation from AR Scorpii through the synchrotron emission process.

\section{Broadband Data Set}

In this work, we use archival results from the radio, infrared, ultraviolet, optical and X-ray observations of AR Scorpii available so far in the literature [14,15]. We have also analyzed data from the Fermi-Large Area Telescope (LAT) observations for a period of more than 10 years to search for $\gamma$-ray emission from AR Scorpii. A brief description of the broadband archival data as well as a detailed analysis of the Fermi-LAT data are given below.

\subsection{Archival Data}

We have obtained the spectral archival points at radio, infrared, ultraviolet, optical and X-ray energies from Figure 4 of Marsh et al. (2016) [14] and Figure 10 of Takata et al. (2018) [15]. These flux measurements in different wavebands include observations from a number of ground and space-based instruments during the period 2006-2016. The X-ray flux points correspond to the time averaged spectrum in the energy range $0.15-12 \mathrm{keV}$ from the XMM-Newton observations of AR Scorpii on September 19, 2016. The XMM-Newton observation covers more than two orbits of AR Scorpii and the X-ray emission shows a large variation over the orbit. A detailed description of the XMM-Newton data can be found in [15]. 


\subsection{Fermi-LAT Data}

The Large Area Telescope (LAT) onboard the Fermi satellite is a pair-conversion detector to measure the energy, arrival time, and direction of the $\gamma$-ray photons in the energy range $30 \mathrm{MeV}$ to more than $1 \mathrm{TeV}$ [21]. It also detects the intense cosmic ray background of the charged particles and radiation trapped at the orbit of the satellite. For $\gamma$-ray photons at $1 \mathrm{GeV}$ energy, the Fermi-LAT has an effective collection area of $\sim 1 \mathrm{~m}^{2}$ at normal incidence, a wide field of view of 2.4 steradians ( $20 \%$ of the sky), and an angular resolution of $0.8^{\circ}$. At lower energies, the photon angular resolution is poor $\left(\sim 5^{\circ}\right.$ at $\left.0.1 \mathrm{GeV}\right)$ and it improves with the increase in energy $\left(\sim 0.1^{\circ}\right.$ above $\left.10 \mathrm{GeV}\right)$. Therefore, the angular resolution of the FermiLAT can be approximated as a power law function of energy $\left(\mathrm{E}^{-0.8}\right.$, where $\mathrm{E}$ is the energy of the $\gamma$-ray photon) in its operational energy range. The whole sky coverage is provided by the Fermi-LAT every three hours in survey mode of its operation.

In this work, we have extracted the Pass 8 Release 3 (P8R3) data for the position of AR Scorpii (RA: $16^{h} 21^{m} 47.28^{s}$, Dec: $-22^{\circ} 53^{\prime} 10.39^{\prime \prime}$ ) collected through the last decade by the Fermi-LAT during August 4, 2008 to March 31, 2019 (MJD 54682-58573) from the Fermi Science Support Centre 1 . The data is processed using standard Fermi Science Tools software version 1.0.1 (Fermi 1.0.1). We have considered only SOURCE class events (evclass $=128$, evtype $=3$ ) corresponding to the P8R3_SOU RCE_V6 instrument response function detected within a circular region of interest (ROI) of $10^{\circ}$ radius centred on the position of AR Scorpii for the above period in the energy range $100 \mathrm{MeV}$ to $500 \mathrm{GeV}$ using the gtselect tool. In addition, a maximum zenith angle cut of $90^{\circ}$ is applied to avoid the albedo contamination from the Earth limb $\gamma$-rays while creating the good time intervals. We have used unbinned maximum-likelihood technique implemented in the gtlike tool to further analyse the dataset. A background model is generated using all the sources reported in the fourth Fermi gamma-ray catalog (4FGL) [22] and recent Galactic diffuse and extragalactic isotropic background emission templates. All the 4FGL point sources present within the ROI are included in the model file for background subtraction keeping their spectral forms the same as defined in the 4FGL catalog [22]. Since the target source AR Scorpii is not reported in the 4FGL catalog, we have manually added this source in the model file with a spectral shape defined by a simple power law function. We use an iterative method to optimize the contribution of sources in the background model file. The associated spectral parameters of all the sources within the ROI including AR Scorpii are left free to vary during the unbinned likelihood fitting. The significance of $\gamma$-ray signal is estimated from the maximum-likelihood test statistic (TS: square-root of TS gives the statistical significance) as defined in [23]. After every fit, we remove the background sources from the model file corresponding to a value of $\mathrm{TS}<25$ (statistical significance $\sim 5 \sigma$ ) and the process is repeated until we get all the background sources

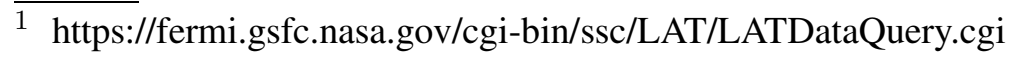




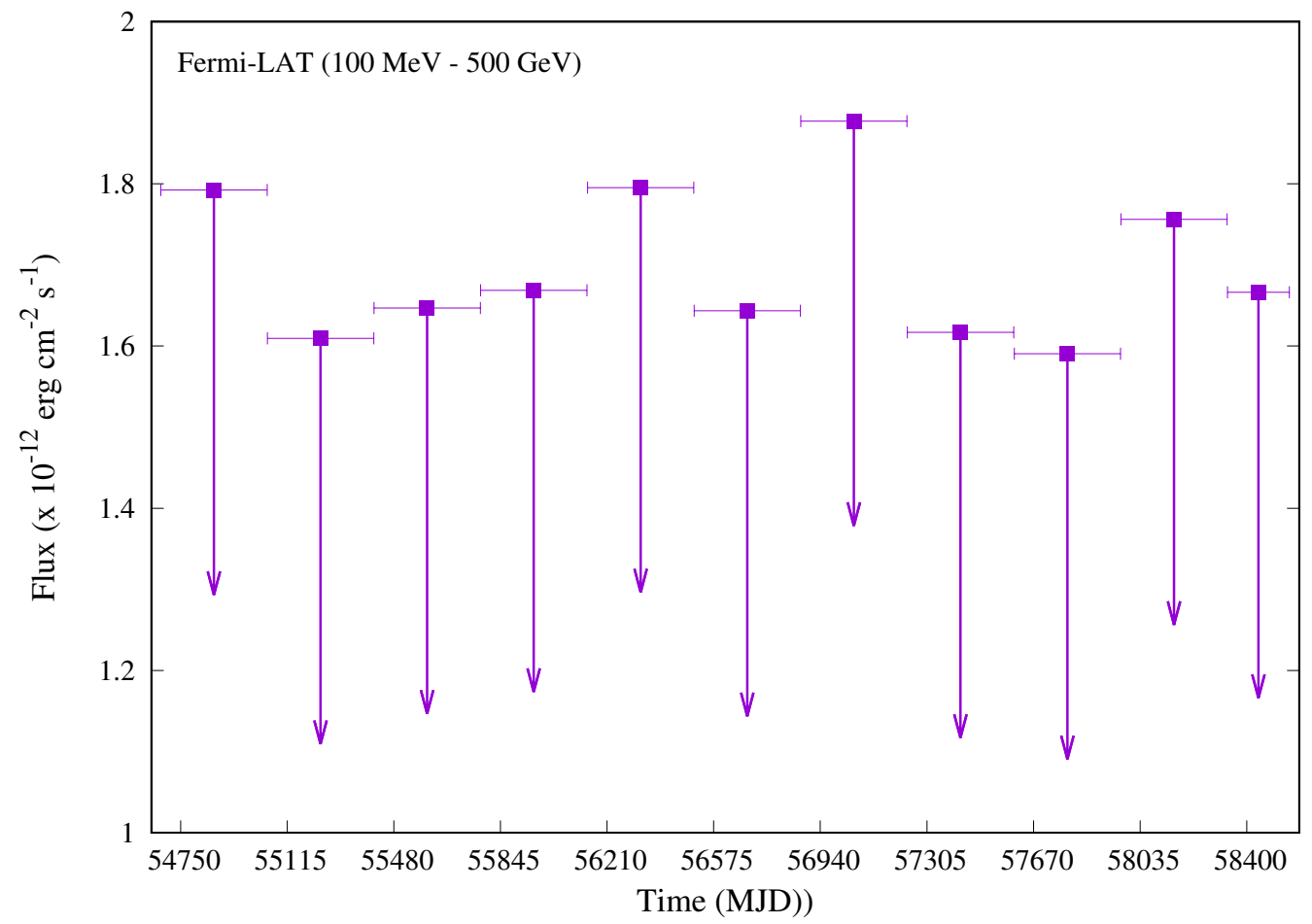

Fig. 1. One year binned (except the last data point which is averaged over about eight months) $\gamma$-ray light curve of the white dwarf binary system AR Scorpii from the Fermi-LAT observations between August 4, 2008 and March 31, 2019 (MJD 54682-58573). The downward arrows indicate $2 \sigma$ upper limit on the integral energy flux above $100 \mathrm{MeV}$.

with TS $\geq 25$ within the ROI in the model file except the target source AR Scorpii. We perform the final unbinned likelihood fitting by allowing the spectral parameters of AR Scorpii and other background sources within $5^{\circ}$ from the center of ROI to vary and the spectral parameters of all other sources lying beyond $5^{\circ}$ are freezed to the values obtained from the optimization.

\section{Results and Discussion}

\subsection{Search for $\gamma$-ray emission from AR Scorpii}

The Pass 8 dataset from the Fermi-LAT observations provides a better measurement of the energy of $\gamma$-ray events over a wider energy range with their improved reconstruction and a significant increase in the effective area at lower energies. From the unbinned-likelihood fitting as described above, we observe that the Fermi-LAT data collected in the direction of AR Scorpii for a period of more than ten years assuming a power law spectral index of -2.7 in the energy range of $100 \mathrm{MeV}-500$ $\mathrm{GeV}$, exhibit a TS value of 8. This implies that the $\gamma$-ray emission from AR Scorpii integrated over a decade from the Fermi-LAT observations is not statistically sig- 
nificant $(<3 \sigma)$ and the ratio of the error in the integral flux to the absolute value is more than 0.5 . Therefore, we have estimated the $2 \sigma$ upper limit of $2.27 \times 10^{-12}$ erg $\mathrm{cm}^{-2} \mathrm{~s}^{-1}$ on the integral energy flux above $100 \mathrm{MeV}$ for the $\gamma$-ray emission from the binary system AR Scorpii. The low statistical significance of the $\gamma$-ray observation from AR Scorpii is consistent with the fact that this source is not reported in the latest 4FGL catalog which includes more than 5000 sources above $4 \sigma$ significance [22]. We have also generated the yearly light curve for AR Scorpii to observe any temporal fluctuation in the $\gamma$-ray emission from this source. The one year binned light curve of AR Scorpii in the energy range $100 \mathrm{MeV}$ to $500 \mathrm{GeV}$ is shown in Figure 1. All the flux points in the light curve (Figure 1) correspond to the $2 \sigma$ upper limit on the integral flux. This indicates that AR Scorpii is not a $\gamma$-ray source above the current detection sensitivity of the Fermi-LAT. The Galactic diffuse emission is bright in the $\mathrm{MeV}-\mathrm{GeV}$ energy regime and provides a dominant contribution to the background radiation near the Galactic plane. Due to a relatively broad angular resolution of the Fermi-LAT at lower energies, the emission from a faint $\gamma$-ray source like AR Scorpii may be contaminated by the diffuse background or photons from the nearby sources. This can affect the search for a pulsed signal from the weak $\gamma$-ray sources. An event weighting technique can be used to search for a pulsation from a faint $\gamma$-ray source [24]. The search for pulsed $\gamma$-ray signal from the WD binary system AR Scorpii is beyond the scope of this work.

\subsection{Broadband Spectral Energy Distribution Modelling}

We have employed a leptonic synchrotron model with two emission components to reproduce the time averaged broadband SED of the binary system AR Scorpii. We assume that the non-thermal radiation from AR Scorpii is produced by the population of relativistic electrons through the synchrotron emission. The electron energy distribution is described by a smooth broken-power law of the form

$$
N(\gamma)=K\left[\left(\frac{\gamma}{\gamma_{b}}\right)^{p}+\left(\frac{\gamma}{\gamma_{b}}\right)^{q}\right]^{-1} ; \gamma_{\min }<\gamma<\gamma_{\max }
$$

where $\gamma=\frac{E}{m_{e} c^{2}}$ is the typical Lorentz factor of the electron with $m_{e}$ being the electron rest mass. $K$ is the normalization coefficient, $p$ and $q$ are the low and high energy electron spectral indices before and after the break respectively. $\gamma_{b}$, $\gamma_{\min }$ and $\gamma_{\max }$ are the Lorentz factors corresponding to the break, minimum and maximum energies of the electrons respectively. The synchrotron emissivity due to an isotropic electron distribution in a tangled magnetic field $(B)$ is calculated as

$$
j_{s y n}(\nu)=\frac{1}{4 \pi} \int_{\gamma_{\min }}^{\gamma_{\max }} N(\gamma) P(\nu, \gamma) d \gamma
$$

where $\nu$ is the frequency of the synchrotron photon and $P(\nu, \gamma)$ is the single electron synchrotron emissivity averaged over an isotropic distribution of pitch angles 


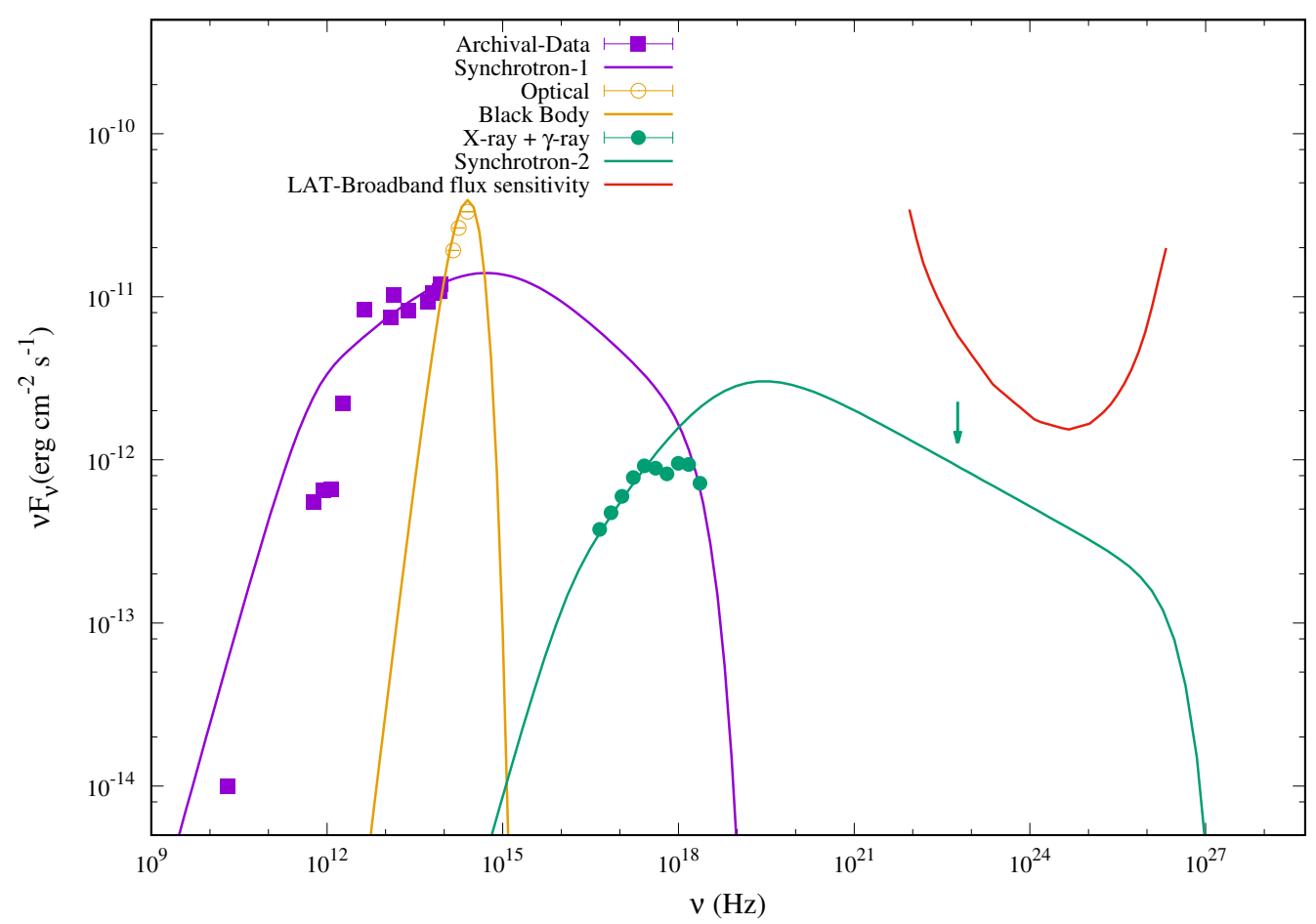

Fig. 2. Broadband spectral energy distribution of AR Scorpii with the time averaged observed data points without measurement errors and model curves. The curves with purple and green colours correspond to the Synchrotron-1 and Synchrotron-2 components of the model for a time averaged non-thermal emission from the binary system AR Scorpii respectively. The yellow curve is associated with the black body emission at a temperature of $T_{b} \sim 3100 \mathrm{~K}$ from the companion M-type star of radius $r=R_{b} \sim 3 \times 10^{10} \mathrm{~cm}$ in the binary system. The data point with downward arrow represent $2 \sigma$ upper limit on the integral flux above $100 \mathrm{MeV}$ from the Fermi-LAT observations over a decade. The measured $2 \sigma$ upper limit and non-thermal emission predicted by the Synchrotron- 2 component of the model indicate that the $\gamma$-ray emission from the source is very weak and below the current flux sensitivity of the Fermi-LAT, depicted by the red curve (taken from the Fermi-LAT Performance at the Fermi Science Support Center).

[25,26]. $P(\nu, \gamma)$ can be expressed as

$$
P(\nu, \gamma)=\frac{\sqrt{3} \pi e^{3} B}{4 m_{e} c^{2}} F\left(\frac{\nu}{\nu_{c}}\right)
$$

with the critical frequency,

$$
\nu_{c}=\frac{3 \gamma^{2} e B}{16 m_{e} c}
$$

where $e$ is the charge of the electron, and $F\left(\frac{\nu}{\nu_{c}}\right)$ is the synchrotron power function, which can be approximately given by [26]

$$
F\left(\frac{\nu}{\nu_{c}}\right) \approx 1.8\left(\frac{\nu}{\nu_{c}}\right)^{1 / 3} \mathrm{e}^{-\frac{\nu}{\nu_{c}}}
$$


The synchrotron power function peaks at $\frac{\nu}{\nu_{c}}=0.29$. This indicates that the peak frequency $\left(\nu_{p}\right)$ in the synchrotron spectrum is about $29 \%$ of the characteristic frequency $\left(\nu_{c}\right)$ defined by Equation 9 . The synchrotron flux received by the observer from the source is given by

$$
F_{o b s}(\nu)=\frac{V}{d^{2}} j_{s y n}(\nu)
$$

where $d$ is the luminosity distance ( $\sim$ distance) of the source and $V$ is the volume of the emission region. Assuming that the emission region is a homogeneous sphere of radius $R_{e m}$ filled with the relativistic electrons defined by Equation 6 and permeated in a tangled magnetic field of strength $B$, we have reproduced the time averaged broadband SED of AR Scorpii. The model curves along with the data points in different wavebands are shown in Figure 2. We observe that the time averaged broadband emission from the binary system AR Scorpii can be broadly reproduced by two non-thermal components referred to as Synchrotron-1 and Synchrotron2. The multi-wavelength data points in Figure 2 have been used without taking into account the measurement errors from different instruments and are reprocduced through the $\chi$-by-eye fitting process. It is evident from Figure 2 that the Synchrotron-1 component of the model describes the emission in the low energy band (radio-IR-Optical-UV), whereas the Synchrotron-2 component predicts soft $\mathrm{X}$-ray and $\gamma$-ray emissions from the binary system AR Scorpii. The high energy end of the X-ray emission (hard X-rays) in the energy range $0.15-12 \mathrm{keV}$ is reproduced by the tail of the synchrotron spectrum from the Synchrotron-1 component of the model. A comparison of the $\gamma$-ray emission level predicted by the Synchrotron2 model with the broadband flux sensitivity of the Fermi-LAT in Figure 2 suggests that the $\gamma$-ray flux above $100 \mathrm{MeV}$ expected from AR Scorpii is below the current detection sensitivity of the LAT as well as the $2 \sigma$ upper limit on the integral flux derived from more than 10 years of observations. The best fit model parameters derived from the non-thermal broadband SED modelling of AR Scorpii are given in Table 1. We note that the size of the emission region $\left(R_{e m}\right)$ for the Synchrotron1 and Synchrotron-2 components is much smaller than the light cylinder radius $\left(R_{L C}\right)$ of the MWD and/or the binary separation $(a)$ in AR Scorpii. This implies that the non-thermal emission zones are situated well inside the light cylinder. The magnetic field strength in the X-ray and $\gamma$-ray emission regions (Synchrotron-2) is much higher than that in the low energy emission zone (Synchrotron-1). However, the strength of the magnetic field in both the emission regions is significantly less than the surface magnetic field $\left(B_{s}\right)$ of the MWD in AR Scorpii. The dipole magnetic field of the MWD at a radial distance $x$ is given by

$$
B_{x}=B_{s}\left(\frac{R}{x}\right)^{3}
$$

Using this relation, we get an estimate of the locations of the emission regions from the MWD as $x_{1}=3.1 \times 10^{10} \mathrm{~cm}$ and $x_{2}=3.1 \times 10^{9} \mathrm{~cm}$ corresponding to the Synchrotron-1 and Synchrotron-2 components respectively. This implies that 
the low energy emission zone (Synchrotron-1) is near to the M-type companion star $\left(x_{1} \sim a \sim 10^{10} \mathrm{~cm}\right)$, whereas the high energy emission zone (Synchrotron-2) is located between the MWD and M-type companion star $\left(x_{2}<a\right)$ in the binary system.

The particle spectral indices $p$ and $q$ describing the smooth broken-power law energy distribution (Table1) indicate an efficient acceleration of electrons to relativistic energies and synchrotron cooling in the binary system AR Scorpii. The energy density of relativistic electrons $\left(U_{e}\right)$ in the emission region is given by

$$
U_{e}=m_{e} c^{2} \int_{\gamma_{\min }}^{\gamma_{\max }} N(\gamma) d \gamma
$$

From Table 1, we find that the energy density of the electrons in the high energy emission zone (Synchrotron-2) is about 5 times higher than that in the low energy emission region (Synchrotron-1). This indicates that the electrons associated with the Synchrotron-2 component are accelerated to higher relativistic energies $\left(\gamma_{\max } \sim 10^{6}\right)$ than those involved in the Synchrotron-1 component. Therefore, electrons near to the MWD experience relatively more acceleration as compared to the far away electrons. The values of $p$ and $q$ for both the emission components are very close to the condition of radiative cooling break $q=p+1$. The synchrotron cooling time scale for an electron is defined as [27]

$$
t_{\text {sync }}=\frac{9}{4} \frac{m_{e}^{3} c^{5}}{e^{4} B^{2} \gamma}=7.7 \times 10^{4}\left(\frac{10 G}{B}\right)^{2}\left(\frac{10^{2}}{\gamma}\right) \text { second }
$$

If the maximum energy of an electron in the emission region is determined by the synchrotron cooling, the acceleration timescale should be the same as $t_{\text {sync }}$. Therefore, assuming $\gamma=\gamma_{\max }$, the acceleration timescale of an electron in AR Scorpii can be expressed as

$$
t_{a c c}=7.7 \times 10^{4}\left(\frac{10 G}{B}\right)^{2}\left(\frac{10^{2}}{\gamma_{\max }}\right) \text { second } .
$$

Using the values of $B$ and $\gamma_{\max }$ from Table 1, we find that $t_{a c c}$ is very small and therefore, suggests a very fast and efficient acceleration of electrons in AR Scorpii. The exact acceleration process for the electrons in the WD binary systems remains unclear. However, several plausible scenarios have been proposed for AR Scorpii. In the first scenario [28], a magnetized plasma ejected from the polar cap of the MWD sweeps the stellar wind from the M-type companion star and interaction between the plasma and stellar wind leads to the formation of a bow shock. This bow shock propagates in the stellar wind and accelerates the electrons to relativistic energies in the wind to produce synchrotron radiation. In the second scenario [29], the magnetic interaction on the surface of the M-type companion star has three dissipation effects namely heating of the surface of M-star, an overflow from the M-star and acceleration of electrons to the relativistic energies with $\gamma=50-100$. The ac- 
Table 1

Best fit model parameters from the non-thermal spectral energy distribution modelling of the binary system AR Scorpii.

\begin{tabular}{lccc}
\hline Parameter & Symbol & Synchrotron-1 & Synchrotron-2 \\
\hline Size of emission region & $R_{e m}$ & $1.8 \times 10^{10} \mathrm{~cm}$ & $1.4 \times 10^{10} \mathrm{~cm}$ \\
Magnetic field strength & $B$ & $1.1 \times 10^{3} \mathrm{G}$ & $1.1 \times 10^{6} \mathrm{G}$ \\
Low energy index of particle ditribution & $p$ & 2.3 & 1.8 \\
High energy index of particle ditribution & $q$ & 3.7 & 3.4 \\
Break energy of particle distribution & $\gamma_{b} m_{e} c^{2}$ & $200 \mathrm{MeV}$ & $715 \mathrm{MeV}$ \\
Minimum Lorentz factor of particle distribution & $\gamma_{\min }$ & 10 & 50 \\
Maximum Lorentz factor of particle distribution & $\gamma_{\max }$ & $2 \times 10^{4}$ & $8 \times 10^{6}$ \\
Particle energy density & $U_{e}$ & $0.46 \mathrm{erg} \mathrm{cm}^{-3}$ & $2.40 \mathrm{erg} \mathrm{cm}^{-3}$ \\
\hline
\end{tabular}

celerated electrons move from the companion star to the surface of the MWD along the magnetic field lines and are trapped in the closed magnetic field lines region due to the magnetic mirror effect. The synchrotron radiation is produced by the trapped electrons at the magnetic mirror point. In the third scenario [30], the particles are accelerated through the magnetic reconnection or Fermi acceleration process in a turbulent collision region produced by the interaction of the MWD magnetic field with a dense atmosphere of the M-type star. In a relatively different hybrid scenario [31], electrons and protons are accelerated to the relativistic energies close to the surface of the companion M-star as postulated in the third scenario and the broadband non-thermal emission is produced by the relativistic electrons and/or by the electron-positron pairs from the pion decay created by the interaction of relativistic protons with the matter in the atmosphere of the M-star. Under the framework of the bow shock acceleration, the electron energy distribution has been reasonably described by a broken-power law with $p \sim 2.4$ and $q=p+1$ constrained by the X-ray observations [28]. The measurements of the optical linear polarization from AR Scorpii support the bow shock acceleration of electrons [16]. Therefore, the electron energy distribution described by a smooth broken-power law in the present study can be attributed to the bow shock acceleration scenario.

However, from Figure 2, we observe that a hump at the optical frequencies cannot be reproduced by the non-thermal synchrotron emission predicted by the Synchrotron1 model. Therefore, we use a simple black body emission to model this optical emission from AR Scorpii. The spectral energy distribution for a thermal emission from a black body is estimated as

$$
\nu F_{\nu}=8 \pi \mathrm{h}\left(\frac{\mathrm{R}_{\mathrm{b}}}{\mathrm{d}}\right)^{2} \frac{\nu^{4}}{\mathrm{c}^{2}}\left(\mathrm{e}^{\mathrm{h} \nu / \mathrm{kT}_{\mathrm{b}}}-1\right)^{-1}
$$


where $R_{b}$ and $T_{b}$ are the radius and temperature of the black body respectively. $h$ and $k$ are the Planck and Boltzmann constants respectively. From the best fit of the optical hump in Figure 2, we get $R_{b}=3.2 \times 10^{10} \mathrm{~cm}$ and $T_{b}=3100 \mathrm{~K}$. These parameters are in good agreement with the values reported in the literature [14,15,28]. Hence, the black body can be associated with the companion M-type star in AR Scorpii. This supports the above finding that the Synchrotron-1 emission zone is near to the M-star in the binary system. Therefore, the thermal emission from the M-type star dominates over the synchrotron radiation at the optical frequencies. The dominant thermal emission from the M-type star can play a major role in the depolarization of the synchrotron radition at the optical wavelengths. Therefore, the thermal contribution of the M-type star should be taken into account while modelling the synchrotron polarization of the source. The intrinsic synchrotron polarization modelling will help to better understand the acceleration process in AR Scorpii.

\section{Conclusions}

We have used the broadband archival data from radio to X-ray observations to reproduce the time averaged spectral energy distribution of the binary system AR Scorpii under the framework of a leptonic emission model. We have also derived a $2 \sigma$ upper limit on the $\gamma$-ray integral flux above $100 \mathrm{MeV}$ by analysing the FermiLAT data for a period of more than a dacade. The important findings of this study are as follows:

- The synchrotron emission produced by the relativistic electrons described by a smooth broken-power law energy distribution from two independent components located well inside the light cylinder of the MWD contributes to the observed broadband SED of AR Scorpii.

- Low energy observations in the radio, infrared, optical and ultraviolet frequency bands are broadly reproduced by the non-thermal emission which originates from a spherical region near the M-type companion star. In this region, we find that the strength of the magnetic field is $\sim 10^{3} \mathrm{G}$ and the electron energy distribution is decribed by the spectral indices 2.3 and 3.7 before and after the break respectively with a break energy of $\sim 200 \mathrm{MeV}$.

- Most of the X-ray flux points in the energy range $0.15-12 \mathrm{keV}$ are reproduced by the synchrotron emission from a spherical zone with a magnetic field strength of $\sim 10^{6} \mathrm{G}$ and located at a distance of $\sim 10^{9} \mathrm{~cm}$ from the MWD. The relativistic electrons in this region also follow an energy distribution with spectral indices 1.8 and 3.4 before and after the break respectively and having a break at $\sim 715$ $\mathrm{MeV}$. The flux points at the high energy end of the X-ray spectra are described by the tail of the synchrotron radiation which is dominant at the low energy component of the broadband SED of AR Scorpii.

- The measured optical emission from AR Scorpii indicates a hump in the broad- 
band SED and cannot be modelled by any of the non-thermal emission components. Instead, the optical hump is well reproduced by a black body spectrum associated with the M-type companion star in the binary system.

- The high energy $\gamma$-ray emission level predicted by the SED modelling of the source is below the $2 \sigma$ upper limit on the integral energy flux above $100 \mathrm{MeV}$ as well as the current broadband flux sensitivity of the Fermi-LAT. This suggests that if the binary system AR Scorpii emits gamma rays, it would be a weak Galactic source, but we cannot make an implicit assumption that it is. However, it can be a potential target for the upcoming ground-based $\gamma$-ray observatory namely the Cherenkov Telescope Array (CTA) with a low energy threshold and better sensitivity.

\section{Acknowledgements}

Authors thank the anonymous reviewer for the important and valuable suggestions to improve the manuscript. We acknowledge the use of public data obtained through the Fermi Science Support Center (FSSC) provided by NASA, and other archival data from various observations in this study.

\section{References}

[1] P. E. Tremblay et al., 2016, MNRAS, 461, 2100

[2] J. D. Cummings et al., 2018, ApJ, 866, 21

[3] L. Ferrario et al., 2015, SSRv, 191, 111

[4] M. J. Darnley et al., 2012, ApJ, 746, 61

[5] P. Szkody et al., 2011, AJ, 142, 181

[6] M. Revnivtsev et al., 2008, A\&A, 489, 1121

[7] B. Zhang \& J. Gil, 2005, ApJL, 631, L143

[8] T. Gold, 1968, Nat, 218, 731

[9] J. Pétri, 2019, MNRAS, 484, 5669

[10] I. Contopoulos \& A. Spitkovsky, 2006, ApJ, 643, 1139

[11] L. Ferrario \& R. Wehrse, 1999, MNRAS, 310, 189

[12] L. Ferrario et al., 1993, MNRAS, 260, 149

[13] S. H. Lubow \& F. H. Shu, 1975, ApJ, 198, 383 
[14] T. R. Marsh et al., 2016, Nat, 537, 374

[15] J. Takata et al., 2018, ApJ, 853, 106

[16] D. A. H. Buckley et al., 2017, NatAs, 1, 0029

[17] J. Takata \& K. S. Cheng, 2019, ApJ, 875, 119

[18] B. Marcote et al., 2017, A\&A, 601, L7

[19] E. R. Stanway et al., 2018, A\&A, 611, A66

[20] P. Garnavich et al., 2019, ApJ, 872, 67

[21] W. B. Atwood et al., 2009, ApJ, 697, 1071

[22] The Fermi-LAT collaboration: S. Abdollahi et al., 2020, ApJS, 247, 33

[23] R. J. Mattox et al., 1996, ApJ, 461, 396

[24] P. Bruel, 2019, A\&A, 622, A108

[25] G. Ghisellini et al., 1988, ApJL, 334, L5

[26] S. Sahayanathan et al., 2018, RAA, 18, 035

[27] Y. Inoue et al., 2019, ApJ, 880, 40

[28] J.-J. Geng et al., 2016, ApJL, 831, L10

[29] J. Takata et al., 2017, ApJ, 851, 143

[30] J. I. Katz, 2017, ApJ, 835, 150

[31] W. Bednarek, 2018, MNRAS, 476, L10 